\title{
Low-Frequency Transcranial Magnetic Stimulation over Left Dorsal Premotor Cortex Improves the Dynamic Control of Visuospatially Cued Actions
}

\author{
Nick S. Ward, ${ }^{1}$ Sven Bestmann, ${ }^{1,2}$ Gesa Hartwigsen, ${ }^{3}$ Michael M. Weiss, ${ }^{3}$ Lars 0. D. Christensen, ${ }^{1}$ \\ Richard S. J. Frackowiak, ${ }^{2,4}$ John C. Rothwell, ${ }^{1}$ and Hartwig R. Siebner ${ }^{3,5}$ \\ ${ }^{1}$ Sobell Department of Motor Neuroscience and ${ }^{2}$ Wellcome Trust Centre for Neuroimaging, University College London, Institute of Neurology, London WC1N 3BG, \\ United Kingdom, ${ }^{3}$ Department of Neurology, Christian-Albrechts University, 24105 Kiel, Germany, ${ }^{4}$ Service de Neurologie, Centre Hospitalier Universitaire Vaudois \\ BH10, 1011 Lausanne, Switzerland, and ${ }^{5}$ Danish Research Centre for Magnetic Resonance, Hvidovre University Hospital, 2650 Hvidovre, Denmark
}

Left rostral dorsal premotor cortex (rPMd) and supramarginal gyrus (SMG) have been implicated in the dynamic control of actions. In 12 right-handed healthy individuals, we applied $30 \mathrm{~min}$ of low-frequency $(1 \mathrm{~Hz})$ repetitive transcranial magnetic stimulation (rTMS) over left rPMd to investigate the involvement of left rPMd and SMG in the rapid adjustment of actions guided by visuospatial cues. After rTMS, subjects underwent functional magnetic resonance imaging while making spatially congruent button presses with the right or left index finger in response to a left- or right-sided target. Subjects were asked to covertly prepare motor responses as indicated by a directional cue presented 1 s before the target. On $20 \%$ of trials, the cue was invalid, requiring subjects to readjust their motor plan according to the target location. Compared with sham rTMS, real rTMS increased the number of correct responses in invalidly cued trials. After real rTMS, task-related activity of the stimulated left rPMd showed increased task-related coupling with activity in ipsilateral SMG and the adjacent anterior intraparietal area (AIP). Individuals who showed a stronger increase in left-hemispheric premotor-parietal connectivity also made fewer errors on invalidly cued trials after rTMS. The results suggest that rTMS over left rPMd improved the ability to dynamically adjust visuospatial response mapping by strengthening left-hemispheric connectivity between rPMd and the SMG-AIP region. These results support the notion that left rPMd and SMG-AIP contribute toward dynamic control of actions and demonstrate that lowfrequency rTMS can enhance functional coupling between task-relevant brain regions and improve some aspects of motor performance.

\section{Introduction}

In an ever-changing world, the need to flexibly adjust our motor plans to sudden changes in the environment is critically important. This includes the ability to generate an appropriate action in response to an external cue, even if the cued response is in conflict with our current motor intentions. Several lines of evidence show that the rostral part of the dorsal premotor cortex (rPMd) is important for action selection, with the left rPMd playing a dominant role (Passingham et al., 1998; Schluter et al., 1998). The rPMd is consistently activated when movement selection relies on spatial or nonspatial visual mapping rules (Kurata et al., 2000; Toni et al., 2002; Amiez et al., 2006; Cavina-Pratesi et al., 2006; Grol et al., 2006; Grafton et al., 2008). The involvement of rPMd in conditional movement selection is more critical if stimulus-

Received Sept. 11, 2009; revised March 4, 2010; accepted May 10, 2010.

This work was supported by the Volkswagenstiftung (H.R.S.) and The Wellcome Trust. N.S.W. was supported by The Wellcome Trust. S.B. was supported by the Biotechnology and Biological Sciences Research Council, United Kingdom. H.R.S. was supported by the Danish Research Council for Health and Disease (Forskningsrådet for Sundhed og Sygdom, Grant 271-08-0669). L.O.D.C. was supported by the Alfred Benzon Foundation, Hellerup, Denmark. J.C.R. was supported by the Medical Research Council, United Kingdom.

The authors declare no competing financial interests.

Correspondence should be addressed to Dr. Hartwig R. Siebner, Danish Research Centre for Magnetic Resonance, Hvidovre University Hospital, Kettegaards Alle 30, 2650 Hvidovre, Denmark. E-mail: hartwig.siebner@drcmr.dk. DOI:10.1523/JNEUROSCI.4499-09.2010

Copyright $\odot 2010$ the authors $\quad 0270-6474 / 10 / 309216-08 \$ 15.00 / 0$ response mapping rules are difficult. For instance, the rPMD is more involved in action selection if the locations of a visual cue and the required response are spatially incompatible (Iacoboni et al., 1998; Dassonville et al., 2001; Schumacher and D'Esposito, 2002) or spatial cues impose specific restrictions on the set of permissible responses (van Eimeren et al., 2006).

The anterior supramarginal gyrus (SMG) and adjacent anterior intraparietal area (AIP) form a key area for controlling grasping movements and hand-object interactions (Culham et al., 2006). Several recent studies that used transcranial magnetic stimulation (TMS) to transiently disrupt neuronal processing in the human SMG-AIP region indicated that it might have a more general role in the on-line control of actions (Tunik et al., 2007). The disruptive effects of TMS showed that the SMG-AIP region is involved in the integration of action goals, efference copy, and sensory inputs (Tunik et al., 2005; Rice et al., 2006). In accordance with this notion, on-line TMS over the left SMG-AIP region delayed finger responses to spatially compatible target stimuli, but only in a nonroutine situation in which a precue prompted subjects to prepare a different movement (Rushworth et al., 2001a). The same region was also found to be activated in a neuroimaging study when subjects covertly attended to their finger movements (Rushworth et al., 2001b).

The left PMd and SMG-AIP regions represent key nodes in the dynamic control of visually guided actions, yet little is known 
about how the relative weighting of these two nodes change dynamically in the context of a transient focal lesion. Here, we applied low-frequency $(1 \mathrm{~Hz})$ repetitive TMS (rTMS) over the left rPMd to induce a mild and transient disruption of neuronal processing in the stimulated PMd (Lee et al., 2003; Siebner et al., 2003; Rounis et al., 2006). After rTMS, participants were studied with whole-brain functional magnetic resonance imaging (fMRI) while they performed a precued reaction time task based on visuospatial cues. We hypothesized that the disruptive effect of 1 $\mathrm{Hz}$ rTMS on left rPMd will change the distributed control of visually guided actions in the left-hemispheric frontoparietal network, including the left SMG-AIP region.

\section{Materials and Methods}

\section{Participants}

Twelve right-handed male volunteers (age, 26-49 years; mean, 32.8), with no history of neurological disorder or head injury, were recruited from the database of volunteers at the Functional Imaging Laboratory, Institute of Neurology, University College London (UCL). Written informed consent was obtained from all participants. The study was approved by the Joint Ethics Committee of the Institute of Neurology, UCL, and National Hospital for Neurology and Neurosurgery, UCL Hospitals NHS Foundation Trust, London.

\section{Transcranial magnetic stimulation}

Each participant underwent real and sham rTMS on 2 separate days, at least $5 \mathrm{~d}$ apart. The order of intervention was counterbalanced across subjects. In each rTMS session, 1800 biphasic stimuli were given over the left rPMd using a MagStim rapid stimulator connected to four booster modules (MagStim Company). According to Rizzo et al. (2004), the rPMd stimulation site was located $2.5 \mathrm{~cm}$ rostrally to the "motor hot spot," defined functionally as the point of maximum evoked motor response in the relaxed right first dorsal interosseus muscle. We chose a premotor site $2.5 \mathrm{~cm}$ rostral to the motor hot spot because in previous neuroimaging studies, peak activations related to conditional movement selection clustered in the rostral portion of the PMd, $18-26 \mathrm{~mm}$ anterior to movement-related activation in the primary motor hand area $\left(\mathrm{M}_{\mathrm{HAND}}\right)$ (for review, see Picard and Strick, 2001).

The rTMS protocol was identical to the protocol used by Siebner et al. (2003) because it resulted in a suppression of neuronal activity in the stimulated rPMd and connected brain regions for at least $1 \mathrm{~h}$ after the end of stimulation. All participants received two 15 min trains of $1 \mathrm{~Hz}$ rTMS separated by an intertrain interval of $1 \mathrm{~min}$, delivered outside the MRI scanner. Stimulation intensity was set to $90 \%$ of individual resting motor threshold (RMT) of the right FDI muscle. The RMT was defined as the lowest stimulus intensity that elicited at least five visible twitches in the right FDI muscle in 10 consecutive stimuli given over the motor hot spot. A standard figure-of-eight-shaped coil (double $70 \mathrm{~mm}$; coil type $\mathrm{P} / \mathrm{N}$ 9925; MagStim Company) was used for real rTMS. For sham rTMS, a specially designed sham coil (MagStim Company) that induced no magnetic field but provided a comparable acoustic stimulus was used. The coil was positioned with the handle at $45^{\circ}$ to the sagittal plane. The current flow of the initial rising phase of the biphasic pulse in the TMS coil induced a current flowing from lateroposterior to medioanterior in the underlying motor cortex. On completion of rTMS, subjects were transported into the MRI scanning suite by wheelchair. The time from the end of the rTMS to the commencement of functional imaging data acquisition was exactly 7 min for all subjects.

The use of a sham coil introduced a control condition that accounted for many unspecific effects such as vigilance, arousal, auditory stimulation, and order effects. The only minor difference that could matter is the lack of tactile sensation with sham TMS. However, we do not consider this to be critical as several minutes elapsed between the end of rTMS conditioning and the start of the fMRI session. An advantage of sham stimulation is that one can exclude any direct stimulation effect on taskrelated brain activity in the sham condition, which is difficult to exclude if one applies the same real TMS protocol over a second brain area. Our sham procedure adopted in the present study was previously used by our group in combined TMS-neuroimaging studies (Lee et al., 2003; Siebner et al., 2003; Rounis et al., 2006) and was judged to be a valuable control option for off-line studies in a recent consensus paper that included more than 30 experts in the field (Siebner et al., 2009).

\section{Experimental paradigm}

During scanning, subjects performed a cued two-choice reaction time task (Rounis et al., 2006). Subjects were asked to visually fixate on a central cross ("+") present throughout the scanning session. The task was to respond to a right- or left-sided target ("+ O" or "O +") appearing for $250 \mathrm{~ms}$ on a screen. Participants were asked to make spatially compatible responses by pressing a button with either the left index finger (in response to the left-sided target) or the right index finger (in response to the right-sided target). Fast but error-free performance was emphasized. The likely position of the target was indicated by a visuospatial cue (" $<+<$ " or " $>+>$ ") lasting $250 \mathrm{~ms}$ and appearing $1000 \mathrm{~ms}$ before the appearance of the target. Cues were valid in $80 \%$ of the trials with respect to the subsequent move cue. All responses were recorded by computer using COGENT Cognitive Interface software (Wellcome Trust Centre for Neuroimaging, London, UK). The data were subsequently analyzed using Matlab 6.0 (Mathworks). For each experimental condition, we calculated the mean reaction times and mean error rates.

Each fMRI session included four different event types: correctly cued right button press (RC), correctly cued left button press (LC), incorrectly cued (i.e., cued to the left) right button press (RI), and incorrectly cued (i.e., cued to the right) left button press (LI). To vary the rate of presentation, null events $(\mathrm{N})$ were included as a trial type during which the central fixation cross was maintained throughout with no presentation of cue or target. There were a total of 660 trials in each fMRI session (132 each of RC and LC, 33 each of RI and LI, and $330 \mathrm{~N}$ trials) with an intertrial interval of $2000 \mathrm{~ms}$. The order of presentation of event types was randomized.

\section{Functional neuroimaging}

A VISION system (Siemens), operating at $2 \mathrm{~T}$, was used to acquire both T1-weighted anatomical images $(1 \times 1 \times 1.5 \mathrm{~mm}$ voxels $)$ and $\mathrm{T} 2{ }^{*}$ weighted MRI transverse echo-planar images (EPIs; matrix, $64 \times 64$; pixel size, $3 \times 3 \mathrm{~mm}^{2}$; echo time, $40 \mathrm{~ms}$ ) with blood oxygenation leveldependent contrast. A total of 620 volumes consisting of $28 \times 5 \mathrm{~mm}$ slices were acquired continuously during each session, with an effective repetition time of $2.15 \mathrm{~s}$ per volume.

Imaging data were preprocessed using Statistical Parametric Mapping (SPM5; Wellcome Trust Centre for Neuroimaging; http://www.fil.ion. ucl.ac.uk/spm/) implemented in Matlab 6 (Mathworks) (Friston et al., 1995a; Worsley and Friston, 1995). All volumes were realigned, unwarped (Andersson et al., 2001), and slice-time corrected. The resulting volumes were then normalized to a standard EPI template based on the Montreal Neurological Institute (MNI) reference brain in Talairach space (Talairach and Tournoux, 1988) and resampled to $2 \times 2 \times 2 \mathrm{~mm}$ voxels. All normalized images were then smoothed with an isotropic 8 $\mathrm{mm}$ full-width half-maximum Gaussian kernel to account for intersubject anatomical differences and allow valid statistical inference according to Gaussian random field theory (Friston et al., 1995c). The time series in each voxel were high-pass filtered at $1 / 128 \mathrm{~Hz}$ to remove low-frequency confounds and scaled to a grand mean of 100 over voxels and scans within each session.

\section{Statistical analysis}

Behavioral data. Using the mean reaction time as a dependent variable, the effects of rTMS on task performance were examined with a three-way repeated-measures ANOVA (SPSS software, version 14). The ANOVA model included the within-subject factors responding hand (two levels, right or left index finger), validity of cue (two levels, validly vs invalidly cued trials), and type of TMS (two levels, real vs sham rTMS). The Greenhouse-Geisser method was used to correct for nonsphericity when appropriate.

Error rates were analyzed separately for validly and invalidly cued trials using nonparametric Wilcoxon signed-rank tests (all reported $p$ values are two-tailed).

fMRI data. The preprocessed fMRI data were analyzed using Statistical Parametric Mapping (SPM5) implemented in Matlab 6 (Friston et al., 
1995b,c; Worsley and Friston, 1995). Statistical analysis of the imaging data was performed in two stages. In the first stage, four event types were defined for each fMRI session: RC, LC, RI, and LI. Each event type was modeled as a delta function with onset coinciding with the appearance of the cue. Covariates were then convolved with a canonical synthetic hemodynamic response function. To make withinsubject comparisons between the post-real rTMS and post-sham rTMS fMRI sessions, a two-session, fixed-effects model was created for each subject. Each session was modeled with the four covariates described above together with a mean (constant) term over scans for each session to model the main effects of session.

The parameter estimates for each covariate resulting from the least mean squares fit of the model to the data were calculated. Statistical parametric maps of the $t$ statistic (SPM $\{t\})$ resulting from linear contrasts of covariates (Friston et al., 1995c) were generated and stored as separate images for each subject. For each subject, we calculated the main effect of each of the three experimental factors (real or sham rTMS, correct or incorrect cue, right or left index finger button press), as well as interactions between them.

The data for the second stage of analysis comprised pooled parameter estimates for each of these contrasts across all subjects in a randomeffects analysis. Contrast images for each subject were entered into a one-sample $t$ test for each contrast of interest. The height threshold for the resulting $\operatorname{SPM}\{t\}$ was set at $p<0.001$, uncorrected for multiple comparisons across whole brain, and the extent (or cluster) threshold was set at $p<0.05$, corrected for multiple comparisons across whole brain. Analysis was performed across the whole brain. In addition, we were particularly interested in two a priori regions of interest (ROIs): the area of left rPMd under the coil, the homologous rPMd in the right hemisphere, and a left AIP region. The left anterior intraparietal sulcus (aIPS) near the SMG (referred to as aIPS-SMG region) was defined as an ROI because a previous TMS study had shown that this area is critically involved in readjusting intended movements in a cued two-choice reaction time task (Rushworth et al., 2001a). ROIs were created to perform small volume corrections to the statistic in these regions and were defined as spheres of $10 \mathrm{~mm}$ radius centered on (1) the average left rPMd coordinate under the coil (see Results), (2) the homologous coordinate in the right hemisphere, and (3) $x=-52, y=-36, z=56$ as described by Rushworth et al. (2001a). All SPM $\{t\}$ were transformed to the unit normal $Z$ distribution to create a statistical parametric map (SPM $\{Z\})$. All $t$ tests performed within SPM were one-tailed.

To make inferences about changes in "functional coupling" between areas, we tested for any regions showing higher coupling with the targeted left rPMd site after real compared with sham rTMS. To do so, we used the well established psychophysiological interaction (PPI) approach, a data-driven analysis that makes minimal assumptions (Friston et al., 1997; Lee et al., 2003). The PPI analysis was first performed at the single-subject level. First, we identified the peak voxel in each subject for the contrast "sham rTMS compared with real rTMS" within the ROI in the left rPMd. Subject-specific ROIs comprising 10-mm-radius spheres centered on their coordinates were created for each of the post-real rTMS and post-sham rTMS sessions, and the first eigenvariate of the fMRI signal during the trials of interest was extracted from each session. We then tested whether regional covariation of task-related signal between the site of stimulation (i.e., the left rPMd) and all other brain voxels was modulated by the type of preceding rTMS (real or sham). The data for the second stage of the PPI analysis comprised the pooled parameter estimates for each single-subject PPI. Contrast images for each subject were entered into a one-sample $t$ test. The height threshold for the resulting $\operatorname{SPM}\{t\}$ was set at $p<0.001$, uncorrected for multiple comparisons across whole brain, and the extent (or cluster) threshold was set at $p<$
B

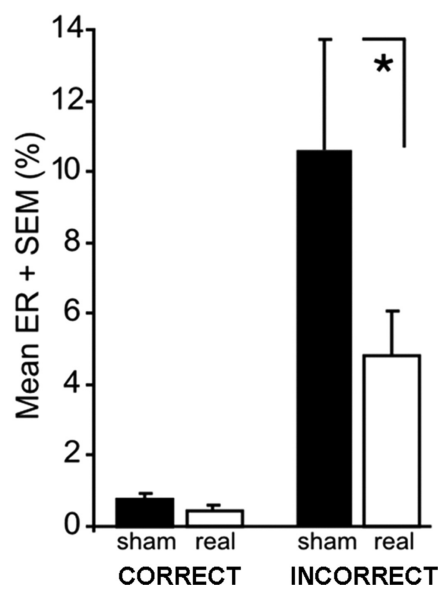
ROIs in the left SMG-AIP region and right rPMd were used for the purpose of small-volume correction (i.e., determining the appropriate statistical threshold for significance), but the PPI analysis was performed using data from all brain voxels.

Anatomical identification was performed by superimposing the maxima of activation foci both on the MNI brain and on the normalized structural images of each subject and by labeling with the aid of the atlas of Duvernoy (1999).

\section{Results}

\section{Behavioral results}

Mean reaction times and error rates are summarized in Figure 1. Reaction times in trials with invalid cues were consistently slower than on validly cued trials (Fig. 1$)\left(F_{(1,11)}=82.10 ; p<0.001\right)$, showing that participants did prepare the cued movements. The responding hand and the type of rTMS had no consistent influence on mean reaction times $(p>0.18)$. ANOVA also revealed no interaction between the factors validity, hand, and type of $\operatorname{rTMS}(p>0.5)$.

Invalid cues increased mean error rates compared with valid cues reflecting the conflict between the cued and the required movement $(Z=5.23 ; p=0.0001)$ (Fig. 1). Real rTMS over left rPMd had an effect on error rates (Fig. 1). Compared with sham rTMS, real rTMS reduced the mean number of errors in invalidly cued trials $(Z=2.00 ; p=0.045)$. For correctly cued trials, a similar trend toward a reduction in error rates after real rTMS was present $(Z=1.70 ; p=0.101)$. Splitting the data for left and right hands revealed no error rate differences between them after sham rTMS $(Z=0.83 ; p=0.590)$ or real rTMS $(Z=0.259 ; p=0.859)$.

\section{Position of the coil}

Inspection of structural T1-weighted scans with TMS surface markers was used to obtain fiducial locations for all 12 subjects. The average location of fiducials adjacent to the left PMd was located at the stereotactic coordinates (mean $\pm \mathrm{SD}$ ): $x=$ $-30.4 \pm 6.9, y=8.2 \pm 3.7, z=66.5 \pm 4.6$.

\section{Imaging results}

A task-related increase in brain activity was seen for all trials (compared to rest) in contralateral primary motor cortex together with a widespread bilateral network of regions including dorsal and ventral premotor cortices, superior and inferior pari- 


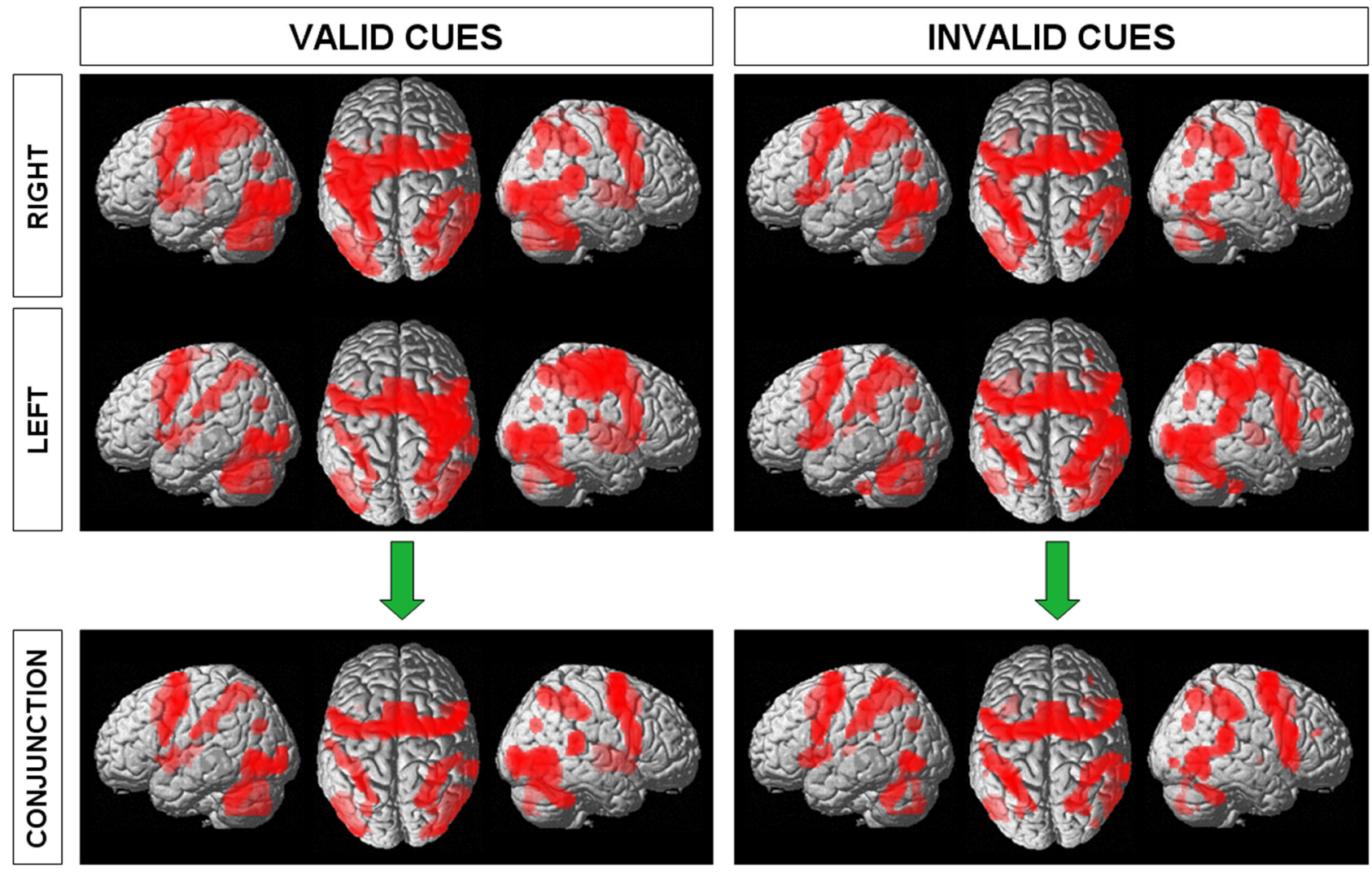

Figure 2. Brain regions active during the cued two-choice reaction time task. Results are shown for right/left hand and validly or invalidly cued trials separately. The conjunction of left and right hands is shown in the bottom panels. SPM $\{Z\}$ is thresholded at $p<0.001$ (uncorrected) for purposes of illustration. Results are rendered onto canonical three-dimensional brains. In each panel, the brain is seen from the left side (left), from above (middle), and from the right side (right).

Table 1. Voxels in which task-related activity was reduced after real compared with sham rTMS to left rostral PMd

\begin{tabular}{|c|c|c|c|c|c|}
\hline \multirow[b]{2}{*}{ Region } & \multirow[b]{2}{*}{ Side } & \multicolumn{3}{|c|}{ Talairach coordinates in MNI space } & \multirow[b]{2}{*}{ Zscore } \\
\hline & & $x$ & $y$ & $z$ & \\
\hline Dorsolateral premotor cortex ${ }^{a}$ & L & -28 & 8 & 64 & 4.04 \\
\hline Ventrolateral premotor cortex & L & -62 & 4 & 20 & 5.12 \\
\hline Inferior frontal gyrus (BA 44) & L & -46 & 16 & 16 & 4.37 \\
\hline Superior temporal sulcus & L & -50 & -36 & -4 & 4.71 \\
\hline Superior parietal cortex & $\mathrm{R}$ & 26 & -40 & 68 & 4.25 \\
\hline
\end{tabular}

Voxels are significant at $p<0.05$, corrected for multiple comparisons across whole brain. R, Right; L, left.

${ }^{a}$ Significant at $p<0.05$, corrected for multiple comparisons within a $10 \mathrm{~mm}$ sphere centered on $x=-30, y=8, z=66$ (estimated coordinates of cortex under coil).

etal cortices, middle occipital gyrus, superior temporal gyrus, putamen, and cerebellar hemispheres (Fig. 2). In particular, taskrelated activity was observed within all three ROIs (left SMGAIP, left and right rPMd). Conjunction analyses revealed that, apart from contralateral sensorimotor cortex and ipsilateral cerebellum, the network activated during this task was bilateral independent of which hand was used during the motor response (Fig. 2).

In keeping with previous findings (for review, see Corbetta and Shulman, 2002), there were relative increases in activity in right inferior parietal cortex $(x=58, y=-30, z=40, Z$ score $=$ 4.24) and ventral premotor cortex $(x=56, y=20, z=22, Z$ score $=4.16)$ for trials with invalid cues compared with trials with valid cues. No brain region showed greater activity during correctly cued trials relative to incorrectly cued trials.

Real compared with sham rTMS resulted in an overall decrease in task-related activity in bilateral ventral premotor cortex as well as left posterior inferior frontal gyrus [Brodmann's area 44
(BA 44)], left superior temporal sulcus, right superior parietal cortex, and left anterior putamen (Table 1), in keeping with our previous results (Siebner et al., 2003). In addition, we found reduced task-related activity after real compared with sham rTMS under the coil in left $\operatorname{rPMD}(x=-28, y=8, z=64, Z$ score $=$ 4.04, $p=0.006$ corrected for multiple comparisons in the left rPMd ROI) and a nonsignificant trend for a similar reduction in left anterior parietal cortex $(x=-54, y=-44, z=52, Z$ score $=$ $3.03, p=0.082$ corrected for multiple comparisons in the SMGAIP ROI). There were no brain regions where real rTMS induced an increase in task-related activity relative to sham rTMS. This remained the case even at a lowered threshold of $p<0.01$, uncorrected for multiple comparisons.

At our prespecified threshold, we found no difference in taskrelated signal reduction after real compared with sham rTMS for trials with valid or invalid cues. However, when looked at separately, there is a clear qualitative difference in the distribution of these 
reductions (supplemental Fig. 1, available at www.jneurosci.org as supplemental material). Real rTMS reduced task-related activity in medial areas (including presupplementary motor area and anterior cingulate sulcus) during valid trials but in a number of lateral areas during invalidly cued trials (including left ventral premotor cortex and middle temporal gyrus, bilateral anterior superior temporal gyri and superior parietal cortices, right inferior parietal cortex and occipital cortex). This particular result is presented for descriptive purposes.

There were no brain regions in which activity showed an interaction between the hand used and either of the other two factors, cue validity or type of rTMS. Thus, outside of contralateral sensorimotor cortex, our results were not influenced by the hand used, and subsequent connectivity analyses were performed using only the factors relating to cue and TMS.

We performed a PPI analysis to investigate whether task-related coupling between the site of stimulation and any other brain region was modulated by real rTMS relative to sham rTMS. In view of our behavioral result, we examined validly and invalidly cued trials separately. We were particularly interested in the effect of rTMS on the left SMG-AIP region shown by a previous TMS study to be important in response generation in the context of invalid cues (Rushworth et al., 2001a). The PPI analysis demonstrated that for invalidly cued trials, greater coupling was seen between the stimulated left premotor site and an area in the left SMG-AIP region $(x=-52, y=-38, z=54, Z$ score $=$ 3.46, $p=0.025$ corrected for multiple comparisons within the left SMG-AIP ROI) after real rTMS compared with sham rTMS (Fig. 3A). There were no significant changes in coupling with any other areas. Furthermore, there were no TMSinduced changes in coupling during the performance of valid trials.

We observed individual variability in the degree to which coupling between left SMG-AIP and left rPMd was strengthened as illustrated by single-subject examples (Fig. 3B). Furthermore, we also observed individual variation in the effect of real rTMS on error rates. We therefore looked for a correlation between real rTMS-induced (1) change in coupling between the stimulated premotor site and other brain regions (using a whole-brain voxel-wise approach) and (2) change in error rates. We found that for invalidly cued trials, error rate reduction correlated with an increase in coupling between the stimulated left rPMd and left SMG-AIP region $(x=-46, y=-36, z=54, Z$ score $=3.56, p=$ 0.025 corrected, $r^{2}=0.68$; and $x=-46, y=-40, z=52, Z$ score $=3.45, p=0.033$ corrected, $r^{2}=0.65$ ) (Fig. 3C). In other words, those individuals who showed a greater rTMS-induced increase in coupling between the stimulated premotor site and left SMG-AIP also showed a greater reduction in errors on inval- idly cued trials. This relationship was independent of the responding hand.

\section{Discussion}

We found that low-frequency rTMS over left rPMd reduced error rates without altering reaction times in a cued two-choice reaction time task when correct response selection was challenged by invalid cues. During invalid trials, participants had to update their motor plan by dismissing the planned response (triggered by the cue) and replace it with the required response (instructed by the target). By asking subjects to perform this task during fMRI, we were able to determine the neural correlates of this change in behavior. Specifically, we found that individual rTMSinduced improvement in error rates correlated with increased intrahemispheric functional coupling between the stimulated left rPMd and SMG-AIP during invalidly cued trials.

The altered premotor-parietal connectivity pattern suggests that $1 \mathrm{~Hz}$ rTMS over left rPMd improved error rates during invalidly cued trials by rendering functional integration between left rPMd and SMG-AIP more efficient. Indeed individual 
changes in premotor-parietal coupling correlated with individual decreases in error rates with invalidly cued trials. This increase in coupling strength was not seen during valid trials, suggesting that the functional premotor-parietal connection is only relevant in nonroutine situations requiring rapid updating of visuospatial response mapping rules.

These findings are important for two reasons. First, premotor-parietal interactions are clearly important during action selection. Our result suggests that the functional integration between rPMd and SMG-AIP in the left hemisphere supports the ability to efficiently update a preexisting motor plan. Second, they lend support to the notion that $1 \mathrm{~Hz}$ rTMS produces its lasting effects on brain function by modifying the connection strength between important nodes of a functional network rather than by local effects on neuronal processing in the stimulated cortex. Critically, $1 \mathrm{~Hz}$ rTMS may enhance functional connectivity between the stimulated cortex and a remote brain area, thereby facilitating a specific brain function.

It might initially seem counterintuitive that the change in coupling we observed occurred independently of the change in taskrelated activity in the two regions, yet there is no reason to assume the two are dependent on one another. Regional activity is thought to reflect intracortical synaptic processing of inputs, whereas a connectivity change indicates a change in the cortical output to a functionally connected brain region. Indeed, another study examining rTMS to left PMd and its effects on action selection have reported that changes in task-related activation and changes in coupling with the stimulated PMd do not occur in the same brain regions (O'Shea et al., 2007). We also want to stress that the alteration in functional connectivity between left rPMd and SMG-AIP is a state-dependent change in the covariation of task-related signal between the two regions but does not assume the presence of direct anatomical connections. Indeed, PMd has a higher probability of connection with the superior parietal lobule rather than anterior inferior parietal cortex (Tomassini et al., 2007), and so it is quite possible that the change in functional connectivity was mediated through another cortical (e.g., ventral premotor cortex) or subcortical area (e.g., anterior putamen).

\section{Integrating preplanned and stimulus-driven response activation}

The invalidly cued trials engaged two competing neural systems, namely preplanned response activation triggered by the precue and direct response activation driven by the instruction cue. The observed improvement in error rate after real rTMS indicates a relative strengthening of stimulus-driven response activation according to an overlearned spatial mapping rule relative to preplanned action selection. With regard to the stimulated PMd, one can assume that PMd is implicated in both types of action selection. It is involved in the generation of predictive feedforward models of sensorimotor control (Christensen et al., 2007), especially when the prediction is based on spatial rules (Schubotz, 2007). These feedforward models are activated by the precue resulting in a preactivation of the precued action. On the other hand, PMd is also involved in applying nonroutine mapping rules, which is necessary when updating a preplanned movement in the context of a new incompatible cue. Left SMG-AIP plays an important integrative role in the on-line control of actions, including rapid updating of motor plans such as stimulus-response mapping rules (Rushworth et al., 2001a; Tunik et al., 2005, 2007; Rice et al., 2006).

We hypothesize then that the behavioral effect we observed with $1 \mathrm{~Hz}$ rTMS over left rPMd resulted from a relative weaken- ing of neuronal circuits subserving a predictive mode of action selection and a relative strengthening of circuits involved in flexible updating of actions. The degree to which this occurred in individual subjects varied, but crucially this was reflected in the magnitude of the increase in functional connectivity between left SMG-AIP and rPMd.

\section{No evidence for compensatory reorganization}

The aftereffects of rTMS in the present study differ from those observed in a recent fMRI-TMS study (O'Shea et al., 2007). It is worth briefly considering this study together with our own to highlight the commonalities and differences. In the study by O'Shea et al. (2007), $1 \mathrm{~Hz}$ rTMS over left PMd increased taskrelated activity in right PMd and medial cortical motor areas during action selection based on complex, arbitrary stimulusresponse mapping rules. Premotor $1 \mathrm{~Hz}$ rTMS had a transient disruptive effect on response selection, but this effect had tapered off before fMRI scanning, and so the increased activity in rightsided motor areas was considered compensatory. Conversely, we found no evidence that $1 \mathrm{~Hz}$ rTMS over left rPMd had a detrimental effect on action selection in our paradigm. In fact, our results suggest that error rate reduction after real rTMS was mediated by increased functional coupling between left rPMd and SMG-AIP rather than compensatory increases in task-related activity elsewhere.

The main differences in protocol between the study by O'Shea et al. (2007) and our own concern the rTMS and the behavioral task. In our study, rTMS was given at higher intensity and for longer and so might have been expected to result in a greater disruptive effect on action selection compared with the protocol used by O'Shea et al. (2007), but this was not the case. Rather, the differences in behavioral task are more likely to be important. The first difference was that O'Shea et al. (2007) asked subjects to perform demanding movement selection tasks involving highly complex nonspatial mapping rules that depend heavily on the integrity of PMd (Schluter et al., 1998). Accordingly, 1 Hz rTMS over left rPMd transiently impaired the subject's ability to perform the task and thus triggered compensatory activity in the nonstimulated right rPMd to restore normal behavior. Conversely, in our study, action selection was simple, involving a highly overlearned spatially congruent response, and so rTMS over left rPMd did not result in compensatory recruitment.

The second important difference is that we presented an informative precue requiring subjects to dynamically update spatially based response selection on appearance of the target cue. In contrast, the response-mapping context was highly stable in the study by O'Shea et al. (2007). Since our participants had to dynamically update visuospatial response selection, we suggest that intrahemispheric premotor-parietal connectivity was highly relevant to task performance in the present study. Thus, $1 \mathrm{~Hz}$ rTMS over left rPMd facilitated dynamic on-line control of actions by improving connectivity between left rPMd and SMG-AIP.

However, there are some important similarities between these studies. Specifically, both found that $1 \mathrm{~Hz}$ rTMS increased functional coupling between the stimulated left rPMd and left hemisphere regions in the context of action selection. In our study, this was with left SMG-AIP, and in the study by O'Shea et al. (2007), it was with posterior intraparietal sulcus and medial motor regions. Neither found an increase in coupling with right-sided regions, in particular PMd. Therefore, one might hypothesize that $1 \mathrm{~Hz}$ rTMS over left PMd alters left-hemispheric frontoparietal connectivity during action selection and that the exact pattern of change in intrahemispheric connectivity depends on 
the mapping rule. Thus, in addition to task-related recruitment of right PMd, the rTMS-induced changes in left-hemispheric connectivity may also have been compensatory in the study by O'Shea et al. (2007).

Together, these two studies show that $1 \mathrm{~Hz}$ rTMS can produce manifold effects on the stimulated functional brain networks. Rather than causing a stereotypic pattern of brain reorganization that generalizes across motor tasks, it is likely that the behavioral consequences as well as short-term reorganization will vary substantially depending on the cognitive processes probed by an experimental task. The results underscore the sensitivity of the off-line TMS-fMRI approach to trace the capacity of functional brain networks to reorganize its cognitive architecture in a context-specific manner.

\section{Nonspecific effects of transcranial stimulation}

One challenge is to control for nonspecific effects of rTMS. Here, we used sham rTMS over left rPMd, which controlled for nonspecific effects such as vigilance, arousal, auditory stimulation, and order effects. Our sham rTMS protocol differed from real rTMS in that it did not involve somatosensory stimulation. Optimized sham TMS coils, which produce somatosensory stimulation, have become available since our study was performed (Rossi et al., 2007). We argue that differences in sensory stimulation at the time of rTMS are unlikely to account for such specific changes in fronto-parietal connectivity after real rTMS because rTMS was applied off-line and $>5$ min before the fMRI session. Furthermore, a nonspecific effect might have been expected to result in a strategy change, leading to longer reaction times accompanying decreases in error rates, which was not observed. An alternative approach would be to apply real rTMS over a second site that would have been preferable in terms of matching somatosensory stimulation but would have introduced other confounds because of the potential effects of rTMS on a different brain region, as well as perceptual differences attributable to differences in the rTMS site (Siebner et al., 2009).

\section{Overall implications}

In conclusion, low-frequency rTMS shapes the function of brain networks in a complex fashion. It appears that an important mechanism of action is to change coupling characteristics between the stimulated and distant brain regions. This is in keeping with work demonstrating that focal $1 \mathrm{~Hz}$ rTMS induces distinct aftereffects in specific intracortical circuits and corticocortical projections (Gilio et al., 2003), which critically depend on the functional state of the cortex at the time of rTMS (Siebner et al., 2004). Our results have two important implications for current applications of $1 \mathrm{~Hz}$ rTMS in both cognitive neuroscience and therapeutic interventions. First, although $1 \mathrm{~Hz}$ rTMS is widely assumed to induce lasting "disruption" of neuronal processing in the stimulated cortex, it might also increase functional coupling between task-relevant brain regions depending on the cognitive state at the time of stimulation. In our study, increased coupling between rPMd and SMG-AIP correlated with improvement in one aspect of motor behavior, although we cannot be sure that others were not adversely affected. Second, $1 \mathrm{~Hz}$ rTMS over M1 is considered to influence pathological interhemispheric interactions after stroke (Hummel and Cohen, 2006), but our results suggest modulation of intrahemispheric, as well as interhemispheric, connectivity as another candidate mechanism by which $1 \mathrm{~Hz}$ rTMS could promote functionally relevant brain reorganization.

\section{References}

Amiez C, Kostopoulos P, Champod AS, Petrides M (2006) Local morphology predicts functional organization of the dorsal premotor region in the human brain. J Neurosci 26:2724-2731.

Andersson JL, Hutton C, Ashburner J, Turner R, Friston K (2001) Modeling geometric deformations in EPI time series. Neuroimage 13:903-919.

Cavina-Pratesi C, Valyear KF, Culham JC, Kohler S, Obhi SS, Marzi CA, Goodale MA (2006) Dissociating arbitrary stimulus-response mapping from movement planning during preparatory period: evidence from event-related functional magnetic resonance imaging. J Neurosci 26:2704-2713.

Christensen MS, Lundbye-Jensen J, Geertsen SS, Petersen TH, Paulson OB, Nielsen JB (2007) Premotor cortex modulates somatosensory cortex during voluntary movements without prioprioceptive feedback. Nat Neurosci 10:417-419.

Corbetta M, Shulman GL (2002) Control of goal-directed and stimulusdriven attention in the brain. Nat Rev Neurosci 3:201-215.

Culham JC, Cavina-Pratesi C, Singhal A (2006) The role of parietal cortex in visuomotor control: what have we learned from neuroimaging? Neuropsychologia 44:2668-2684.

Dassonville P, Lewis SM, Zhu XH, Ugurbil K, Kim SG, Ashe J (2001) The effect of stimulus-response compatibility on cortical motor activation. Neuroimage 13:1-14.

Duvernoy HM (1999) The human brain: surface, three-dimensional sectional anatomy with MRI, and blood supply. Wien, New York: Springer.

Friston KJ, Ashburner J, Frith CD, Poline J-B, Heather JD, Frackowiak RSJ (1995a) Spatial registration and normalization of images. Hum Brain Mapp 3:165-189.

Friston KJ, Holmes AP, Poline JB, Grasby PJ, Williams SC, Frackowiak RS, Turner R (1995b) Analysis of fMRI time-series revisited. Neuroimage 2:45-53.

Friston KJ, Holmes AP, Worsley KJ, Poline J-B, Frith CD, Frackowiak RSJ (1995c) Statistical parametric maps in functional imaging: a general linear approach. Hum Brain Mapp 2:189-210.

Friston KJ, Buechel C, Fink GR, Morris J, Rolls E, Dolan RJ (1997) Psychophysiological and modulatory interactions in neuroimaging. Neuroimage 6:218-229.

Gilio F, Rizzo V, Siebner HR, Rothwell JC (2003) Effects on the right motor hand-area excitability produced by low-frequency rTMS over human contralateral homologous cortex. J Physiol 551:563-573.

Grafton ST, Schmitt P, Van Horn J, Diedrichsen J (2008) Neural substrates of visuomotor learning based on improved feedback control and prediction. Neuroimage 39:1383-1395.

Grol MJ, de Lange FP, Verstraten FA, Passingham RE, Toni I (2006) Cerebral changes during performance of overlearned arbitrary visuomotor associations. J Neurosci 26:117-125.

Hummel FC, Cohen LG (2006) Non-invasive brain stimulation: a new strategy to improve neurorehabilitation after stroke? Lancet Neurol 5:708-712.

Iacoboni M, Woods RP, Mazziotta JC (1998) Bimodal (auditory and visual) left frontoparietal circuitry for sensorimotor integration and sensorimotor learning. Brain 121:2135-2143.

Kurata K, Tsuji T, Naraki S, Seino M, Abe Y (2000) Activation of the dorsal premotor cortex and pre-supplementary motor area of humans during an auditory conditional motor task. J Neurophysiol 84:1667-1672.

Lee L, Siebner HR, Rowe JB, Rizzo V, Rothwell JC, Frackowiak RS, Friston KJ (2003) Acute remapping within the motor system induced by lowfrequency repetitive transcranial magnetic stimulation. J Neurosci 23:5308-5318.

O'Shea J, Johansen-Berg H, Trief D, Gobel S, Rushworth MF (2007) Functionally specific reorganization in human premotor cortex. Neuron 54:479-490.

Passingham RE, Toni I, Schluter N, Rushworth MF (1998) How do visual instructions influence the motor system? Novartis Found Symp 218:129141; discussion 141-146.

Picard N, Strick PL (2001) Imaging the premotor areas. Curr Opin Neurobiol 11:663-672.

Rice NJ, Tunik E, Grafton ST (2006) The anterior intraparietal sulcus mediates grasp execution, independent of requirement to update: new insights from transcranial magnetic stimulation. J Neurosci 26:8176-8182. Rizzo V, Siebner HR, Modugno N, Pesenti A, Munchau A, Gerschlager W, 
Webb RM, Rothwell JC (2004) Shaping the excitability of human motor cortex with premotor rTMS. J Physiol 554:483-495.

Rossi S, Ferro M, Cincotta M, Ulivelli M, Bartalini S, Miniussi C, Giovannelli F, Passero S (2007) A real electro-magnetic placebo (REMP) device for sham transcranial magnetic stimulation (TMS). Clin Neurophysiol 118:709-716.

Rounis E, Stephan KE, Lee L, Siebner HR, Pesenti A, Friston KJ, Rothwell JC, Frackowiak RS (2006) Acute changes in frontoparietal activity after repetitive transcranial magnetic stimulation over the dorsolateral prefrontal cortex in a cued reaction time task. J Neurosci 26:9629-9638.

Rushworth MF, Ellison A, Walsh V (2001a) Complementary localization and lateralization of orienting and motor attention. Nat Neurosci 4:656-661.

Rushworth MF, Krams M, Passingham RE (2001b) The attentional role of the left parietal cortex: the distinct lateralization and localization of motor attention in the human brain. J Cogn Neurosci 13:698-710.

Schluter ND, Rushworth MF, Passingham RE, Mills KR (1998) Temporary interference in human lateral premotor cortex suggests dominance for the selection of movements. A study using transcranial magnetic stimulation. Brain 121:785-799.

Schubotz RI (2007) Prediction of external events with our motor system: towards a new framework. Trends Cogn Sci 11:211-218.

Schumacher EH, D’Esposito M (2002) Neural implementation of response selection in humans as revealed by localized effects of stimulus-response compatibility on brain activation. Hum Brain Mapp 17:193-201.

Siebner HR, Filipovic SR, Rowe JB, Cordivari C, Gerschlager W, Rothwell JC, Frackowiak RS, Bhatia KP (2003) Patients with focal arm dystonia have increased sensitivity to slow-frequency repetitive TMS of the dorsal premotor cortex. Brain 126:2710-2725.

Siebner HR, Lang N, Rizzo V, Nitsche MA, Paulus W, Lemon RN, Rothwell JC (2004) Preconditioning of low-frequency repetitive transcranial magnetic stimulation with transcranial direct current stimulation: evidence for homeostatic plasticity in the human motor cortex. J Neurosci 24:3379-3385.

Siebner HR, Bergmann TO, Bestmann S, Massimini M, Johansen-Berg H, Mochizuki H, Bohning DE, Boorman ED, Groppa S, Miniussi C, PascualLeone A, Huber R, Taylor PC, Ilmoniemi RJ, De Gennaro L, Strafella AP, Kähkönen S, Klöppel S, Frisoni GB, George MS, et al. (2009) Consensus paper: Combining transcranial stimulation with neuroimaging. Brain Stim 2:58-80.

Talairach J, Tournoux P (1988) Co-planar stereotaxic atlas of the human brain. 3-Dimensional proportional system: an approach to cerebral imaging. New York: Thieme.

Tomassini V, Jbabdi S, Klein JC, Behrens TE, Pozzilli C, Matthews PM, Rushworth MF, Johansen-Berg H (2007) Diffusion-weighted imaging tractography-based parcellation of the human lateral premotor cortex identifies dorsal and ventral subregions with anatomical and functional specializations. J Neurosci 27:10259-10265.

Toni I, Shah NJ, Fink GR, Thoenissen D, Passingham RE, Zilles K (2002) Multiple movement representations in the human brain: an event-related fMRI study. J Cogn Neurosci 14:769-784.

Tunik E, Frey SH, Grafton ST (2005) Virtual lesions of the anterior intraparietal area disrupt goal-dependent on-line adjustments of grasp. Nat Neurosci 8:505-511.

Tunik E, Rice NJ, Hamilton A, Grafton ST (2007) Beyond grasping: representation of action in human anterior intraparietal sulcus. Neuroimage 36 [Suppl 2]:S77-S86.

van Eimeren T, Wolbers T, Munchau A, Buchel C, Weiller C, Siebner HR (2006) Implementation of visuospatial cues in response selection. Neuroimage 29:286-294.

Worsley KJ, Friston KJ (1995) Analysis of fMRI time-series revisitedagain. Neuroimage 2:173-181. 